Behavioral/Cognitive

\title{
Brightness-Color Interactions in Human Early Visual Cortex
}

\author{
Dajun Xing, ${ }^{1}$ Ahmed Ouni, ${ }^{2}$ Stephanie Chen, ${ }^{2}$ Hinde Sahmoud, ${ }^{2}$ James Gordon, ${ }^{2,3}$ and Robert Shapley ${ }^{2}$ \\ ${ }^{1}$ State Key Laboratory of Cognitive Neuroscience and Learning \& IDG/McGovern Institute for Brain Research, Beijing Normal University, Beijing 100875, \\ People's Republic of China, ${ }^{2}$ Center for Neural Science, New York University, New York, New York 10003, and ${ }^{3}$ Psychology Department, Hunter College, \\ New York, New York 10065
}

The interaction between brightness and color causes there to be different color appearance when one and the same object is viewed against surroundings of different brightness. Brightness contrast causes color to be desaturated, as has been found in perceptual experiments on color induction and color-gamut expansion in human vision. However, it is not clear yet where in the cerebral cortex the brightness-color interaction that causes these major perceptual effects is located. One hypothesis is that brightness and color signals are processed separately and in parallel within the primary visual cortex V1 and only interact in extrastriate cortex. Another hypothesis is that color and brightness contrast interact strongly already within V1. We localized the brightness-color interaction in human V1 by means of recording the chromatic visual-evoked potential. The chromatic visual-evoked potential measurements decisively support the idea that brightness- color interaction arises in a recurrent inhibitory network in V1. Furthermore, our results show that the inhibitory signal for brightness- color interaction is generated by local brightness contrast at the boundary between target and surround, instead of by the luminance difference between the interior of the color target and its large background.

Key words: brightness; color; cVEP; inhibition; visual cortex; visual perception

\section{Introduction}

The interaction between brightness and color can change the color appearance of an object very markedly. For example, an object is most colorful, most saturated with color, when there is color contrast between the object and its surroundings but also when there is no brightness contrast. Perceived color saturation can be washed out almost completely when there is a large brightness contrast between an object and its surroundings. Examples of the power of increased brightness contrast to reduce color saturation are given in Figure $1 A$. Figure $1 A$ (middle, red and green rectangles) are approximately equally luminant with their surroundings and therefore are maximally colorful. The same color rectangles placed on the black surroundings in Figure $1 \mathrm{~A}$ (bottom) are much less colorful. As demonstrated in Figure $1 \mathrm{~A}$ (top), the effect of brightness contrast is mainly happening at the edges of objects; in Figure $1 A$ (top), we added thin black bands around the edges of the color targets placed on surroundings equal in luminance to the rectangles, and the color was almost as desaturated as on the full black surround in the bottom panel.

\footnotetext{
Received Sept. 8, 2014; revised 0ct. 30, 2014; accepted Nov. 18, 2014

Author contributions: D.X., J.G., and R.S. designed research; D.X., A.O., S.C., H.S., J.G., and R.S. performed research; D.X., J.G., and R.S. contributed unpublished reagents/analytic tools; D.X., A.O., S.C., J.G., and R.S. analyzed data; D.X., J.G., and R.S. wrote the paper.

This work was supported by National Key Basic Research Program of China 2014CB846101, National Natural Science Foundation of China Grant 31371110, the Fundamental Research Funds for the Central Universities, the United States National Institutes of Health Grants T32 EY-07158 and R01 EY-01472, the United States National Science Foundation Grant 0745253, and the Swartz Foundation fellowships.

The authors declare no competing financial interests.

Correspondence should be addressed to Dr. Dajun Xing, State Key Laboratory of Cognitive Neuroscience and Learning \& IDG/McGovern Institute for Brain Research, Beijing Normal University, No. 19, XinJieKoWai ST, HaiDian District, Beijing, 100875, People's Republic of China. E-mail: dajun_xing@bnu.edu.cn.

DOI:10.1523/JNEUROSCI.3740-14.2015

Copyright $\odot 2015$ the authors $\quad 0270-6474 / 15 / 352226-07 \$ 15.00 / 0$
}

Such brightness-color effects can be observed in natural images; for instance, Figure $1 B$ shows that the color appearance of natural visual objects, such as flowers, can be strongly affected by brightness contrast with their surroundings. Recently, precise psychophysical experiments were designed to measure the desaturation of human color perception by brightness contrast (Faul et al., 2008; Bimler et al., 2009); we performed similar experiments (see below).

However, it is not clear yet where in the cerebral cortex such perceptually significant brightness-color interaction takes place. One hypothesis, the classical view of color and brightness derived from ideas about color encoding, originally proposed by Hering in the 19th century (Hurvich and Jameson, 1957), is that brightness and color signals are processed separately and independently and in parallel (Fig. 2A) in early vision and only interact at some high perceptual level well beyond the primary visual cortex (V1). Another hypothesis is that color and brightness contrast interact as early as V1 (Fig. 2B) (Johnson et al., 2008). Here we show that the second hypothesis is correct by means of recording the human chromatic visual-evoked potential (cVEP). The behavior of cVEP from V1 decisively supports the idea that brightness-color interaction arises in a recurrent network in V1, in an inhibitory manner. Furthermore, the results provide direct electrophysiological evidence that color-brightness interactions take place very locally at the edges between a color target and its surroundings. Recurrent inhibition in local cortical circuits has been called a canonical cortical computation, and here we show that this canonical computation implemented in V1 cortex has a strong influence on color perception.

\section{Materials and Methods}

All observers in both psychophysics and EEG experiments gave informed consent to participate in this study. The experiments were conducted in 
A

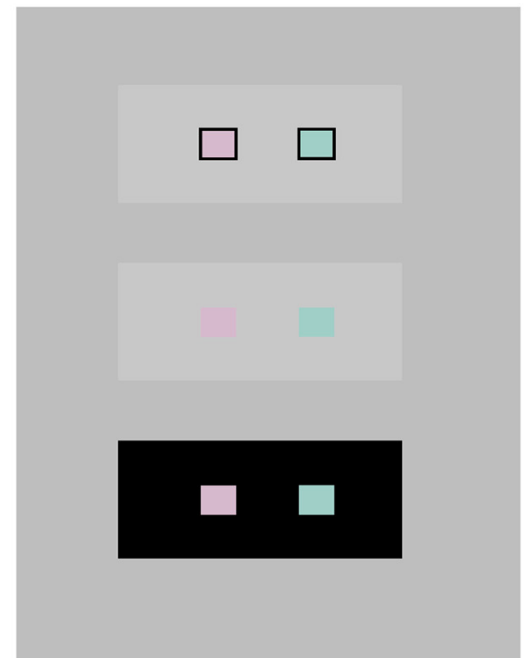

B

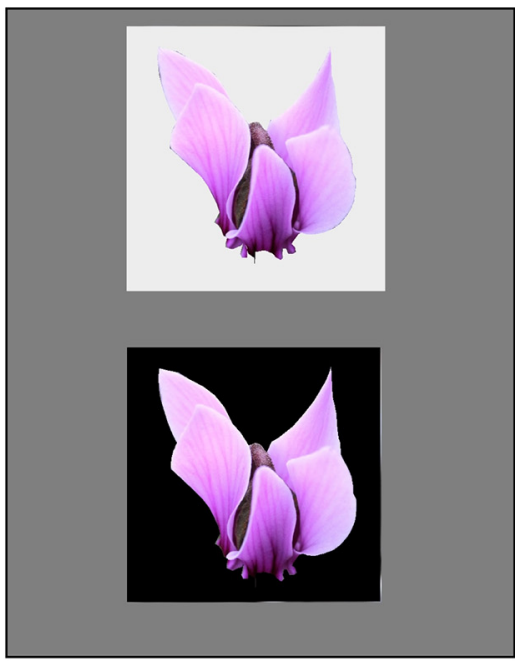

Figure 1. A, Color appearance is strongly affected by surrounding context. The red targets all have the same physical wavelength spectrum, but the perceived color saturation is greatest when brightness contrast between target and local surround is near zero in the middle panel; likewise for the green targets. In addition, a thin black line surrounding the color target strongly affects the color appearance as is apparent in the top. $\boldsymbol{B}$, Color appearance of natural images also is greatly influenced by luminance contrast between target and surroundings. An image of a cyclamen flower, copied from the Internet, placed on a light gray background that is approximately equiluminant with the flower image (above) and on a black background (below), to illustrate the effect of luminance contrast on color appearance of the flower's petals.

A

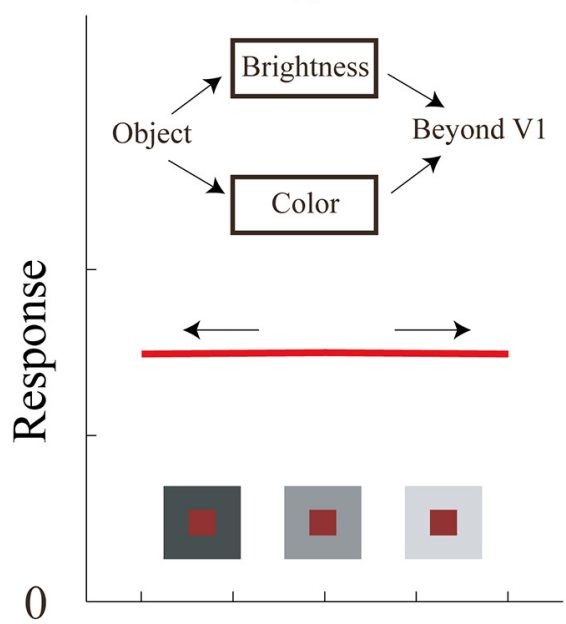

0
B

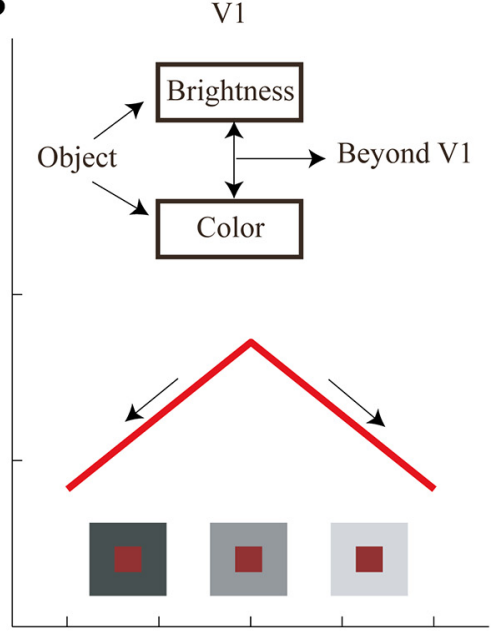

0

\section{Luminance difference}

Figure 2. Two hypotheses for brightness: color interaction and their response prediction. $\boldsymbol{A}$, Demonstration of the parallelchannels hypothesis. According to this hypothesis, the population responses should be constant regardless of the brightness contrast. $\boldsymbol{B}$, Demonstration of the mutual-inhibition hypothesis. According to this hypothesis, the population responses should be decreasing as the brightness contrast (luminance difference) deviates from zero.

accordance with the principles embodied in the Declaration of Helsinki and were approved by the Hunter College/City University of New York and the New York University Institutional Review Boards.

\section{Psychophysics experiments}

Participants. Five observers (four females and one male between the ages of 20 and 23) participated in this experiment. Color vision was assessed with Ishihara plates, and all observers were color normal.

Apparatus. Square $1.15^{\circ}$ foveal chromatic target stimuli with a luminance of $20 \mathrm{~cd} / \mathrm{m}^{2}$ were surrounded contiguously by achromatic surrounds. Surround luminances ranged from 0 to $40 \mathrm{~cd} / \mathrm{m}^{2}$. All stimuli

were presented using a Dell p780 monitor set at a refresh rate of $100 \mathrm{~Hz}$. The full screen display was comprised of $1025 \times 768$ pixels. The screen was viewed at a distance of $1 \mathrm{~m}$. Stimuli were calibrated with a Photo Research Spectrascan 670 spectroradiometer. Red and green chromatic stimuli (dominant wavelengths of 612 and $535 \mathrm{~nm}$ ) were each presented at a medium value of color excitation purity (CEP $=$ 0.15). CEP is defined as the quotient of the distance in CIE $x y$ color space from the white point to the stimulus, divided by the distance from the white point to the spectrum locus of the stimulus' dominant wavelength.

Saturation scaling procedure. We used five surround luminances $(0,10,20,30$, and $40 \mathrm{~cd} /$ $\mathrm{m}^{2}$ ); note that the $20 \mathrm{~cd} / \mathrm{m}^{2}$ surround luminance was equiluminant to the $20 \mathrm{~cd} / \mathrm{m}^{2}$ chromatic stimuli. Participants used saturation scaling to indicate their perception of the relative color saturation of the color target when it was located on the different surrounds. The saturation scaling technique, first introduced by Hurvich and Jameson $(1957,1959)$, asks the observer to describe the percentage of the total sensation (achromatic plus chromatic) that can be attributed to color. In other words, a total absence of hue would be indicated by $0 \%$ saturation, whereas a target that is completely saturated and lacking of any achromatic sensation would be $100 \%$ saturation. Saturation scaling has been shown to be a rapid and reliable method for comparing relative hue quantity (Gordon et al., 1994). The chromatic stimuli were each rated five times in each of the five surrounds. Stimuli were presented for $3 \mathrm{~s}$ with a $20 \mathrm{~cd} / \mathrm{m}^{2}$ gray background on during the 15-20 intertrial interval. This procedure minimized carryover sensory effects. Scaling values for five repeats of each presentation were averaged.

\section{cVEP experiments}

Participants. Thirteen observers (eight males and five females), ranging in age from 17 to 70 years (mean age $=32$ years, median $=23$ years), participated in the cVEP experiments. Data from one of the 13 subjects were excluded for further analysis due to bad quality of VEP signals. Another subject only participated in the cVEP experiment for the color red. The participants in the cVEP experiments were different from those in the psychophysics experiments. Color vision was assessed with HRR Pseudo-Isochromatic Plates. All observers were color normal. Observers had normal (at least 20 of 25) or corrected-to-normal visual acuity at the viewing distance of $114 \mathrm{~cm}$.

Stimuli. A Venus system (Neuroscientific) was used for presenting stimuli and recording electrophysiological responses. A Nokia monitor (Multigraph $447 \times, 90 \mathrm{~Hz}$ frame rate) set to a mean luminance of $20 \mathrm{~cd} / \mathrm{m}^{2}$ was used as a stimulus display. The display size was $20 \times 20 \mathrm{~cm}$, giving a field size of $10 \times 10^{\circ}$ at the viewing distance of $114 \mathrm{~cm}$. Targets were viewed binocularly and were calibrated with a Photo Research PR670 Spectrascan Radiometer/Photometer.

The stimulus pattern was a set of $131^{\circ}$ squares: one square at fixation was surrounded by a circular array of 12 squares. The center of each peripheral square was set $4^{\circ}$ from the center of the central square. The luminance of all squares was $20 \mathrm{~cd} / \mathrm{m}^{2}$. In each experiment, the color 
Table 1. CIE $x y$ values and the color excitation purities of the red and green stimuli used in Figure 4

\begin{tabular}{llll}
\hline & $x$ & $y$ & Excitation purity \\
\hline Reds & 0.4587 & 0.3373 & 0.4024 \\
& 0.4050 & 0.3423 & 0.2406 \\
& 0.3814 & 0.3411 & 0.1690 \\
& 0.3606 & 0.3388 & 0.1055 \\
Greens & 0.3349 & 0.3360 & 0.0272 \\
& 0.2986 & 0.5764 & 0.5948 \\
& 0.3012 & 0.5312 & 0.4841 \\
& 0.3092 & 0.4634 & 0.3170 \\
& 0.3135 & 0.4011 & 0.1647 \\
& 0.3236 & 0.3305 & 0.0125 \\
\hline
\end{tabular}

squares were modulated (rectangular wave in time) periodically at a temporal frequency of $0.5 \mathrm{~Hz}$. Each stimulus cycle consisted of a transition from gray to equiluminant color $(0.5 \mathrm{~s}$ on $)$ and a transition back to gray (color temperature $5800^{\circ} \mathrm{K}$, degrees Kelvin; $20 \mathrm{~cd} / \mathrm{m}^{2} ; 1.5 \mathrm{~s}$ on). The $2 \mathrm{~s}$ period was repeated 30 times for run durations of $60 \mathrm{~s}$. In a given run, all stimulus squares were the same color. CIE $x y$ values of the red and green stimuli (dominant wavelengths of 614 and $543 \mathrm{~nm}$, respectively) and their CEPs are given in Table 1. Both red and green color points are on straight lines in CIE space that run from the white point to their respective dominant wavelengths on the CIE spectrum locus. The equivalent color temperature of the white point was $5800^{\circ} \mathrm{K}$ (degrees Kelvin). In each run, the $10^{\circ} \times 10^{\circ}$ background in which the checks were embedded was set to one of the following luminance values: $0,10,20,30$, and $40 \mathrm{~cd} / \mathrm{m}^{2}$. In the experiments for Figure 6 , the stimulus squares were surrounded by a thin black band $\left(6^{\prime}\right)$ separating them from the gray surround.

cVEP recording. Gold-cup electrodes were placed along the midline of the scalp according to the international ten-twenty system (Klem et al., 1999): active electrode at $\mathrm{Oz}$ (near the occipital pole), reference electrode at $\mathrm{Cz}$ (vertex of the head), and ground electrode at $\mathrm{Pz}$ (midway between $\mathrm{Oz}$ and $\mathrm{Cz}$ ). The EEG, recorded in synchrony with the stimulus, was amplified $(20,000)$, bandpass filtered $(0.1-100 \mathrm{~Hz})$, digitized at a rate of $180 \mathrm{~Hz}$ (two samples per frame of the Nokia display), and stored in a computer for analysis.

Analysis. Responses were averaged across the 30 presentations. The cVEP usually had a single negative going wave followed in some observers by a positive rebound (Crognale, 2002; Crognale et al., 2013). Response strength was quantified by calculating the rms power of the response waveform around the baseline, averaged over the $0.5 \mathrm{~s}$ period of visual stimulation.

\section{Results}

\section{Psychophysics}

Because it is so important for color perception, we sought to replicate prior results by Faul et al. (2008) and Bimler et al. (2009) on color appearance on neutral backgrounds of different brightnesses. Color saturation scaling was performed to quantify the degree of saturation change induced by increasing amounts of luminance contrast. In these trials, observers rated the relative saturation of the chromatic target while the target was displayed on one of five achromatic backgrounds. The chromatic targets appeared red or green on neutral backgrounds. They remained a fixed chromaticity and luminance throughout the trials.

We found that, when a color target appeared with an achromatic surround that had a high luminance contrast with the target, the apparent saturation of the color was decreased. Thus, we replicated the result that, as luminance contrast increased (when either the luminance of the surround increases or decreases relative to the luminance of the target), the apparent color saturation of the target gradually decreased (Faul et al., 2008; Bimler et al., 2009). Figure 3 shows the average of the normalized ratings of

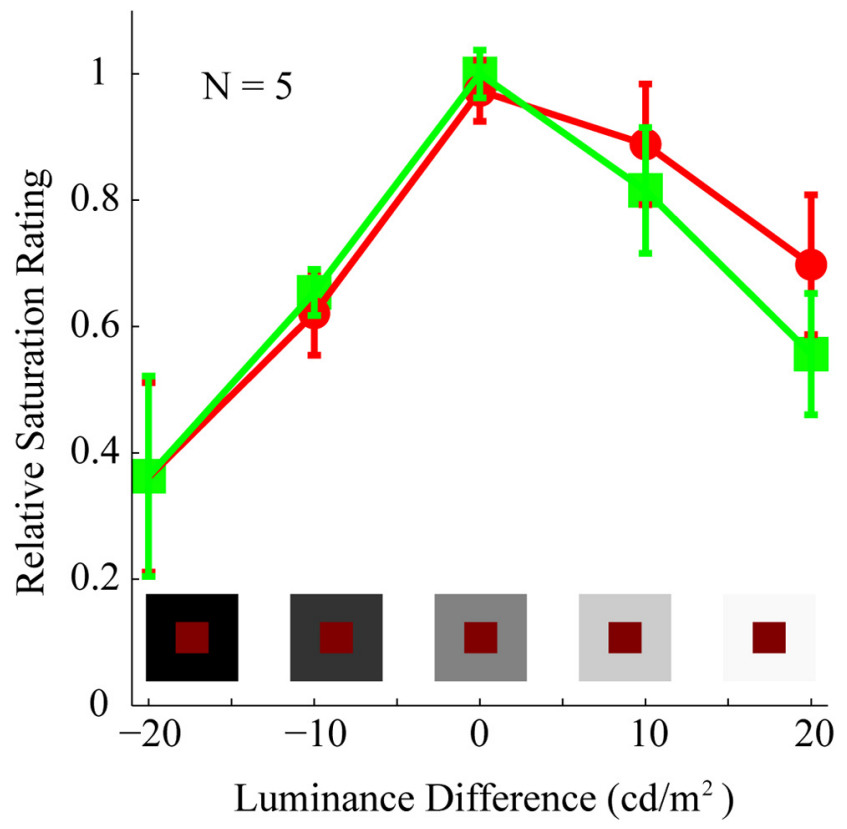

Figure 3. Psychophysics of color suppression by luminance contrast. Group average $(N=5$ observers) of saturation scaling of a target's color as a function of luminance contrast between the target and the neutral surround. The color of the data points and connecting curves represent the color used in the experiments (red and green, respectively).

color saturation of red and green targets (see Methods and Materials). It is evident that maximum color saturation was perceived when luminance contrast was minimal. An average of $21 \%$ luminance contrast in either the negative or positive direction produced $25 \%$ reduction of the scaled color-saturation value.

\section{cVEPs and color cells in V1}

We investigated the link between perceived color saturation and responses of neurons in the visual cortex by using VEPs evoked by the onset of pure-color stimuli in the human EEG, the cVEP, to reveal brain mechanisms of color vision (Murray et al., 1987; Rabin et al., 1994; Crognale, 2002; Souza et al., 2008; Martinovic et al., 2011; Crognale et al., 2013). Evidence that the cVEP reflects V1 activity is as follows. Prior work on VEPs as we recorded them, over occipital cortex along the midline, indicates that the signals recorded come from V1 cortex (Onofrj et al., 1995; Nakamura et al., 2000; Kulikowski et al., 2002). The cVEP does not vary with attention, a result that strongly suggests it is evoked early in cortical visual processing (Highsmith and Crognale, 2010). Furthermore, normal cVEPs have been recorded in cases of cerebral achromatopsia where color appearance was lost and lesions were observed in ventromedial extrastriate cortex, but V1 responses to color were unaffected by the lesion (Victor et al., 1989; Crognale et al., 2013). The combined evidence from source localization, lack of attentional effects, and cerebral achromatopsia indicates that the cVEP is an index of V1 responses to color.

Many investigators have found that color computations in V1 are based on the activity of two kinds of cone-opponent neurons: single- and double-opponent cells (for review, see Shapley and Hawken, 2011). Single-opponent cells respond to large areas of color. Double-opponent cells respond to color patterns, textures, and color boundaries (Johnson et al., 2001; Schluppeck and Engel, 2002; Friedman et al., 2003). The VEP responses we measured were time-locked to the color modulation of a target that was constant in luminance at all times. The equiluminant color modu- 
A

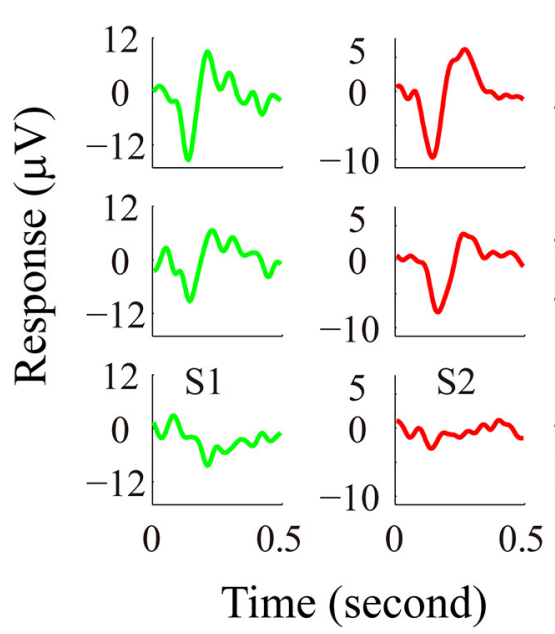

C

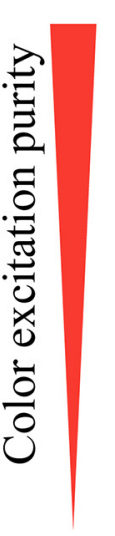

0.3

0

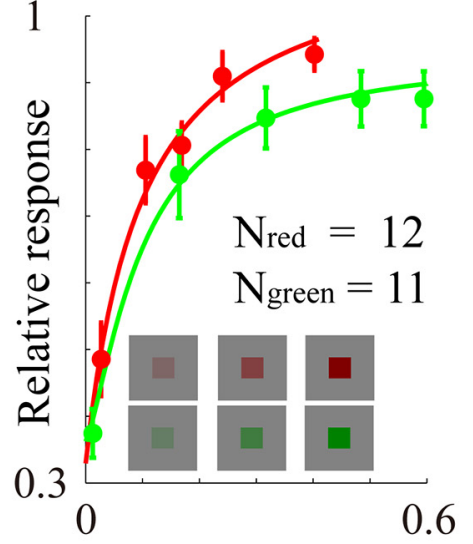

Color excitation purity

Figure 4. The CVEP grows with color excitation purity. CEP is defined as distance in xy color space from the white point to the stimulus relative to the distance from white point to the spectrum locus of the stimulus' dominant wavelength. $A$, The waveforms of an observer's CVEPs to green color targets of different CEPs. Top, CEP $=0.59$. Bottom, CEP $=0.01$. $\boldsymbol{B}$, The waveforms of another observer's cVEPs to red color targets of different purities. Top, CEP $=0.40$. Bottom, CEP $=0.03$. C, Population-averaged magnitudes of CVEPs as functions of red and green CEP. Vertical bars represent SEM. The data were fitted by Naka-Rushton functions.

lation of targets in our experiments produced cVEPs that must have been influenced by single- and double-opponent cells because they are the only V1 cells that respond to color modulation (Johnson et al., 2001; Schluppeck and Engel, 2002). To allow comparisons across observers and to use the entire cVEP signal not just the peak, we used the rms power of the cVEP waveform over the $0.5 \mathrm{~s}$ of stimulus presentation as a measure of cVEP amplitude. cVEPs were graded in amplitude with color saturation (color excitation purity) of the stimuli (Fig. 4), confirming earlier results (Crognale, 2002; Souza et al., 2008). In Figure 4, the population average is taken from the data of 11 of 13 participants for green and 12 of 13 participants for red (the 13th subject was excluded because of bad quality of the VEP signals). The 11 participants whose data are included the averages are those of the 13 who ran in all stimulus conditions, and we only obtained cVEP data for red from the 12th observer. The gradation of cVEP amplitude with the color purity of stimuli for which there was no luminance modulation supports the idea that the cVEP represents the population response of color-sensitive neurons in $\mathrm{V} 1$.

\section{Neuronal mechanisms and competing hypotheses}

Previous psychophysical work (Gordon and Shapley, 2006; Faul et al., 2008; Bimler et al., 2009) suggested that the explanation of the color-brightness phenomenon illustrated in Figure 1 is that the cortical mechanisms for color and brightness perception are mutually inhibitory. Specifically, we hypothesized that, at the neural representation of edges in the cerebral cortex, there is mutual inhibition between neural mechanisms for color and brightness in a "winner-take-all" network (Fig. 2B) (Gordon and Shapley, 2006). Perception is ruled by the strongest signal for boundary identification. The perceptual importance of edges is indicated in Figure $1 A$ (top) where a black band drawn around the targets greatly reduces the saturation of the color targets on the approximately equiluminant surround (Faul et al., 2008).

There is an alternative to the mutual-inhibition hypothesis: the parallel-channels hypothesis (Fig. 2A). Suppose that color saturation is encoded as the population activity profile across all V1 cells (Wachtler et al., 2003). The alternative hypothesis assumes that there is no interaction between color-responsive and colornonresponsive (achromatic) V1 neurons; these two groups form parallel, independent channels (Livingstone and Hubel, 1984). The parallel-channels hypothesis is a good description of color processing in the thalamus (in the lateral geniculate nucleus) where parvocellular and magnocellular channels are literally in parallel (Kaplan, 2014). The parallel-channels hypothesis assumes that the population code in V1 is read out in higher cortical areas (McKeefry and Zeki, 1997; Hadjikhani et al., 1998; Mullen et al., 2007). For instance, the explanation of the appearance of the color targets in Figure 1, according to the parallel-channels view, is that, when brightness contrast increases, V1 colorblind nonopponent cells at the edge boundaries become more activated without affecting the responses to color contrast by V1 double-opponent cells. The resulting population profile then would resemble that produced by a target with lower chroma and would be read out at a higher cortical level as lower saturation. However, population coding also is compatible with the mutual-inhibition hypothesis; the net result of $\mathrm{V} 1$ brightness-color interactions could affect the population distribution of activity within V1 that is interpreted at higher cortical levels.

Our experiments decided between the mutually inhibitory and parallel-channels hypotheses by measuring the response of the color-selective population with cVEPs. The parallel-channels theory predicts that the cVEPs we measured should not change with increasing brightness contrast between target and surround (Fig. 2A). The mutual-inhibition hypothesis predicts a drop in cVEP amplitude with increasing brightness contrast (Fig. 2B). There was a drop in cVEP amplitude for both positive and negative brightness contrast in 12 of 13 observers (see Materials and Methods) we studied, clearly supporting the mutual-inhibition hypothesis. Representative cVEP waveforms from two observers illustrate the drop in cVEP amplitude with brightness contrast (Fig. 5A). Plots of cVEP amplitude versus brightness contrast between color target and surround, averaged across observers. (We only included the 11 subjects who participated in cVEP experiments for both red and green color.) One of the 12 included observers only participated in the cVEP experiment for red (Figure $5 B$ ). Consistently, the largest cVEP was obtained when brightness contrast between target and surround was zero. These results are highly consistent with the prediction of the mutualinhibition hypothesis: color responses are inhibited by brightness strength. Furthermore, our observations of suppression of the cVEP recorded with posterior electrodes indicate that the effect was mainly in V1 because, if V1 responses were unaffected and instead there was suppression in V4 or in LOC cortex, we would not expect to see such large reductions in the cVEP.

\section{Importance of edges}

The perceptual importance of neuronal activity near the edges of targets (Krauskopf, 1963; Faul et al., 2008) is illustrated in Figure $1 A$ (top) by the reduced perceived color saturation of targets on 
equiluminant surrounds when the targets have thin black bands drawn around them. We found analogous reductions of cVEPs when observers viewed colormodulated targets that had black bands around them (Fig. 6), even when the surround outside the black band was equiluminant with the target. This experiment was run on two observers (Fig. 6) who were members of the larger group that contributed data to Figure 5.

\section{Discussion}

The strong effect of brightness contrast on color appearance has been studied previously with human psychophysics. For instance, it has long been known that brightness contrast affects color induction from a colored surround into an achromatic target (Kirschmann, 1891). Kirschmann's third Law stated that chromatic induction was greatest when brightness contrast was minimal. Our own psychophysical measurements of color induction, based on the same technique of saturation scaling used in the present paper, confirmed that perceived color saturation in chromatic induction was maximal when brightness contrast was minimal (Gordon and Shapley, 2006). A very different kind of experiment also suggests that there is a suppressive effect of brightness contrast on color: masking with luminance gratings can raise the threshold of detectability of pure-color gratings (Switkes et al., 1988). The phenomenon we studied in this paper is the effect of brightness contrast on the color appearance of colored targets as in Figure 1. This phenomenon is related to Kirschmann's third Law, but it is different in the following way. In color induction experiments, the target is a neutral color (mid-gray). The region surrounding the target is colored and induces a complementary color in the target. In the experiments in this paper, the targets were colored regions surrounded by regions that were neutral in color (different gray levels from black to white). But as in color induction, the appearance of color was subdued when there was brightness contrast at the boundary between target and surroundings (Fig. 1).

Another important effect on color appearance is the phenomenon of gamut expansion, discovered by Brown and MacLeod (1997; see also Brou et al., 1986). Gamut expansion means that the color saturation of a target is much greater when it is viewed on an equiluminant uniform gray surround than when the same color target is surrounded by a high-contrast checkerboard of colored squares. In a very powerful set of experiments, Faul et al. (2008) found that the phenomenon of color gamut expansion discovered by Brown and MacLeod (1997) was caused mainly by brightness contrast at the borders of the color targets with their surroundings and that color contrast near to or remote from the

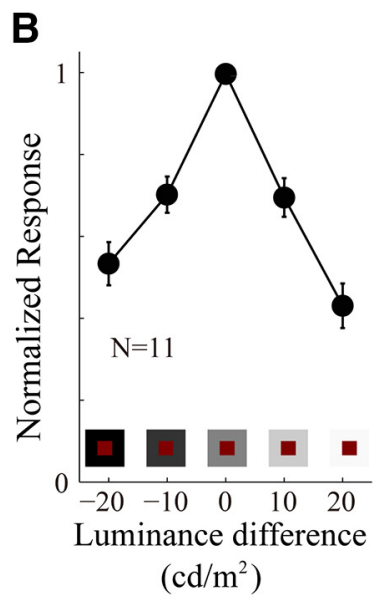

Figure 5. CVEP reduced by increased brightness contrast between color target and its achromatic surround. $\boldsymbol{A}$, The waveforms of two observers' cVEPs to red color targets (luminance was set at $20 \mathrm{~cd} / \mathrm{m}^{2}$ ) with different surround luminances. Left, The bas set at $10 \mathrm{~cd} / \mathrm{m}^{2}$, and the background luminance on the right was set at $30 \mathrm{~cd} / \mathrm{m}^{2}$. Middle, The equiluminance condition; both target and background had luminance of $20 \mathrm{~cd} / \mathrm{m}^{2}$. B , cVEP reduced by increased brightness contrast between color target and achromatic surround - population average. Over 11 observers, magnitudes of cVEPs were CVEP was weaker when brightness contrast between target and background increased. This is true for both red and green color
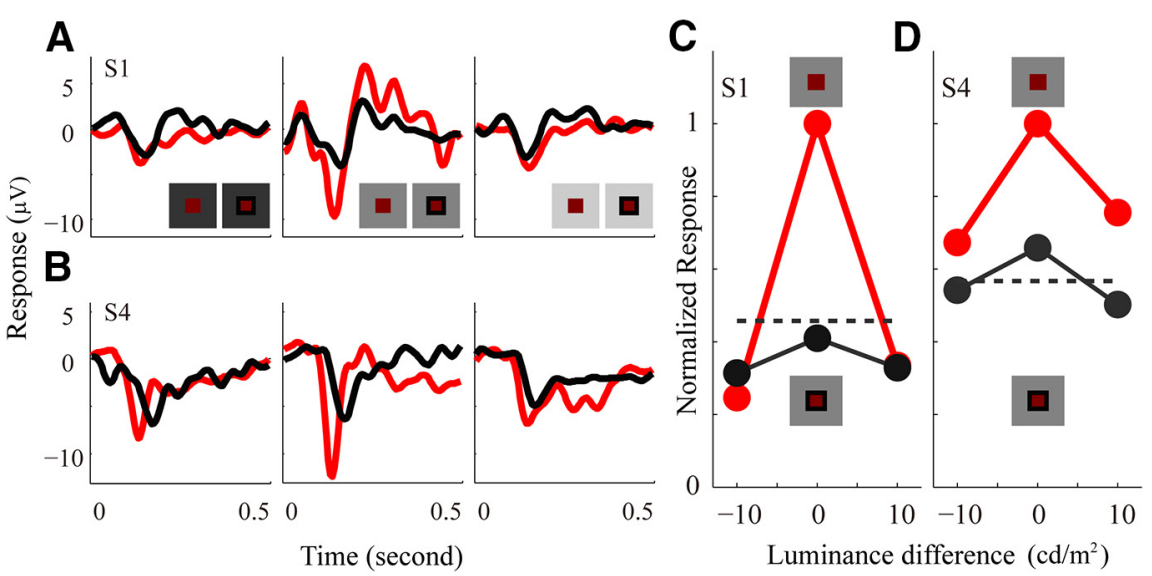

Figure 6. The effect of brightness context on the CVEP was strongly affected by a thin black line $\left(6^{\prime}\right)$ surrounding the color target. $\boldsymbol{A}$, The waveforms of an observer's CVEPs to a red target (luminance, $\left.20 \mathrm{~cd} / \mathrm{m}^{2}\right)$ with different surround luminances $(10,20$, and $30 \mathrm{~cd} / \mathrm{m}^{2}$ ) were strongly affected by a thin $\left(6^{\prime}\right)$ black surrounding line. Waveforms without black line are drawn in red, and those with black line are drawn in black. $\boldsymbol{B}$, The result from another observer. $\boldsymbol{C}, \boldsymbol{D}$, Summarization of the change of cVEP magnitudes before and after a thin line was added around the color target. Black dashed line indicates the CVEP magnitude when surround luminance was set at $0 \mathrm{~cd} / \mathrm{m}^{2}$.

target contributed little to gamut expansion. Thus, gamut expansion and the desaturation by surrounds of high brightness contrast (as in Fig. 1) are probably the same phenomenon. Bimler et al. (2009) studied brightness-color interactions with scaling methods and found results essentially the same as what we show in Figure 3. The results on cVEPs in this paper were designed to uncover the neural locus and mechanism of brightness-color interactions in human vision.

Our experimental data on cVEPs (Figs. 5 and 6) indicate that the mechanism of perceptual brightness color interaction is inhibition in human primary visual cortex.

These data support the mutual-inhibition explanation of brightness-color interactions in perception (for the statement of the mutual-inhibition hypothesis, see Results). It is also possible, even likely, that the parallel-channels hypothesis (also presented 
A

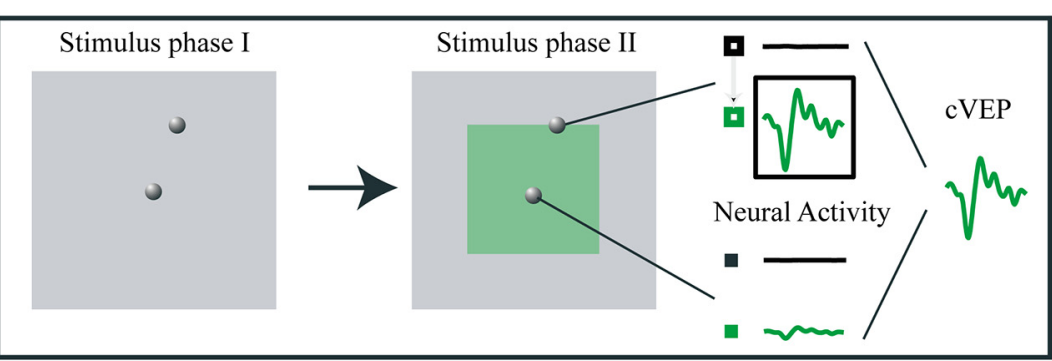

B

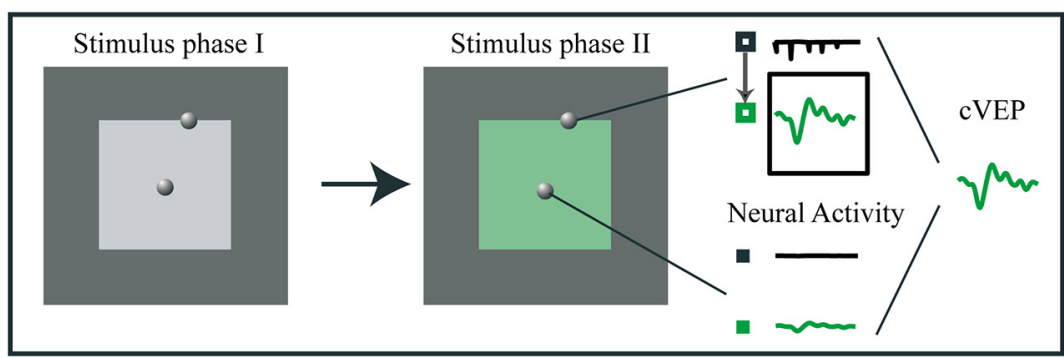

C

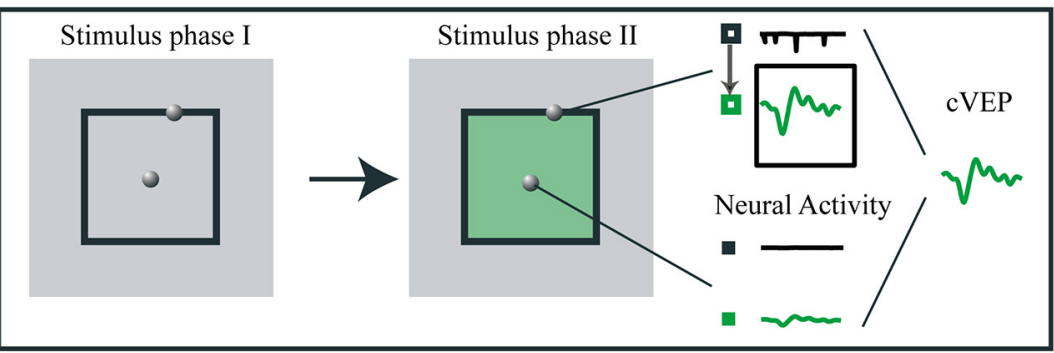

- Luminance Neurons on edges - Color-Lum Neurons on edges

- Luminance Neurons on surface

- Color-Lum Neurons on surface

Figure 7. Proposed neural mechanism of luminance-color suppression. In the CVEP experiment, the V1 population that contributes to the CVEP is comprised of the Color-Lum neurons at the border of the square. $\boldsymbol{A}-\boldsymbol{C}$, Color-Lum neurons' stimuluslocking responses are shown as green curves surrounded by black frames. When the stimulus has the same luminance as its surround, Lum neurons on the edge are inactive $(\boldsymbol{A})$, and there is no suppression of the full-size CVEP. However, when a spatial luminance contrast exists at the square border due to either luminance difference between square and its surround $(\boldsymbol{B})$ or high spatial frequency line ( $($ ), Lum neurons are activated by the stimulus due to asynchronous modulation (e.g., small eye movements or brain state fluctuation). $B, C$, Lum neurons' responses are locked to eye movement but not to stimulus; therefore, Lum neurons' responses ( $\boldsymbol{B}, \boldsymbol{C}$, spikes) cannot be seen in the trial-averaged CVEP. However, such asynchronous Lum neuron responses provide a source of constant inhibition onto Color-Lum neurons. $B, C$, cVEPs are also mainly caused by Color-Lum responses; butbecause Lum neurons are activated, Color-Lum responses are suppressed by inhibition from Lum neurons, and the amplitudes of cVEPs are reduced.

perceptual experiments (Alpern, 1964). Alpern found that simultaneous brightness contrast was reduced by color contrast. We think it is reasonable to hypothesize that Alpern's results could be explained by the mutual inhibition of brightness-responsive neurons by color-responsive neurons, also occurring early in the visual system, in V1. This topic should be the target of future research.

The suppressive effect on color appearance of brightness contrast near the edge of a color target has been shown to cause other perceptual effects, such as the gamut expansion effect (Brown and MacLeod, 1997; Faul et al., 2008). Our results show that these perceptual effects are mediated by corticocortical inhibition early in the visual cortex. Corticocortical inhibition has been found to have an important function in visual cortex (Shapley and Xing, 2013) and in other sensory cortices (Wu et al., 2008; Franks et al., 2011; Isaacson and Scanziani, 2011). Throughout the cortex, neurons engage in a recurrent inhibitory computation to determine what is the most likely sensory information in the environment. Figure 1 illustrates that cortical computation in action in the effect of brightness context on color.

\section{Proposed neural mechanism of luminance-color suppression}

Our results strongly suggest that luminance-color suppression mainly takes place at the border of the stimulus. Here we propose a simple explanation (Fig. 7). V1 neurons can be categorized into three groups (Johnson et al., 2001). One group of neurons are only interested in luminance contrast (Lum-Neuron); another group of neurons are only interested in pure color difference (Color-Neuron); the third group of neurons are interested in both color and

in Results) is also partially correct and that there is population coding of color in V1 and in extrastriate cortex (Wachtler et al., 2003; Shapley and Hawken, 2011). The mutual-inhibition hypothesis is not incompatible with population coding; the net result of brightness-color interactions will shift the peak of the population distribution of activity away from the color-responding population and toward the brightness-responsive, nonopponent (Johnson et al., 2001) population. Such a shift in population distribution will be interpreted at higher cortical levels as less color. The inhibition from brightness onto color signals in V1 revealed in Figure 5 would in this way work together with population coding of color to create the desaturated appearance of color targets on brighter or darker surrounds, as in Figure 1.

The data we have presented in this paper are consistent with the idea that there is inhibitory suppression of the color-responding population by nonopponent cells that respond to brightness contrast. The converse (the mutual inhibition of brightness responding neurons by color-responsive neurons) has so far only been studied in luminance contrast (Color-Lum-Neuron) (Johnson et al., 2001; Li et al., 2014). Generally, Color neurons are the minority in the V1 population, and they respond best at low spatial frequency (Johnson et al., 2001; Schluppeck and Engel, 2002; Conway and Livingstone, 2006). Based on the spatial frequency tuning of the cVEP (Rabin et al., 1994), we infer that Color neurons do not contribute very much to the population activity (cVEP). Also, because it is not clear whether the responses of "Color-Neuron" are suppressed by brightness, we think that the color-luminance (double-opponent) cells are the most likely candidates for brightness-color suppression. Let us only consider Lum neurons and Color-Lum neurons. In our experiment, when a square alternates its color but keeps its luminance constant, V1 neurons whose receptive fields are on the square's surface will not generate strong responses (Fig. 7) because V1 neurons (both Lum neurons and Color-Lum neurons) are tuned to medium spatial frequency and respond poorly to very low spatial frequency, such as a uniform surface (Johnson et al., 2001; Schluppeck and 
Engel, 2002). Cells that mainly respond to the alternation of a square's color are those at the border of the square (Friedman et al., 2003). At the border, Color-Lum neurons' responses strongly synchronize to the alternation of the square color, but Lum neurons respond poorly to the color alternation due to a lack of luminance change. However, Lum neurons can be driven by the stimulus due to asynchronous modulation, such as brain state fluctuations or small eye movements, when spatial luminance contrast exists at the square border (Fig. $7 B, C$ ). Because the fluctuations are not synchronized with color alternation of the square, Lum neurons' responses are invisible in trial-averaged cVEP. But the asynchronous Lum neurons' responses play an important role in color-luminance interaction: they provide the source of a constant suppression onto ColorLum neurons. When there is no spatial luminance contrast (Fig. 7A), Lum neurons are inactive. Therefore, Color-Lum neurons simply respond to color alternation without any suppression originating from Lum neurons. But when there is a spatial contrast (Fig. $7 B, C$ ), the color-locking responses of Color-Lum neurons are modulated by a constant suppression (Johnson et al., 1998) that originates from Lum neurons' asynchronous responses to luminance contrast.

\section{References}

Alpern M (1964) Relation between brightness and color contrast. J Opt Soc Am 54:1491-1492. CrossRef

Bimler DL, Paramei GV, Izmailov CA (2009) Hue and saturation shifts from spatially induced blackness. J Opt Soc Am A Opt Image Sci Vis 26:163-172. CrossRef Medline

Brou P, Sciascia TR, Linden L, Lettvin JY (1986) The colors of things. Sci Am 255:84-92. CrossRef Medline

Brown RO, MacLeod DI (1997) Color appearance depends on the variance of surround colors. Curr Biol 7:844-849. CrossRef Medline

Conway BR, Livingstone MS (2006) Spatial and temporal properties of cone signals in alert macaque primary visual cortex. J Neurosci 26:1082610846. CrossRef Medline

Crognale MA (2002) Development, maturation, and aging of chromatic visual pathways: VEP results. J Vis 2:438-450. CrossRef Medline

Crognale MA, Duncan CS, Shoenhard H, Peterson DJ, Berryhill ME (2013) The locus of color sensation: cortical color loss and the chromatic visualevoked potential. J Vis 13:pii15. CrossRef Medline

Faul F, Ekroll V, Wendt G (2008) Color appearance: the limited role of chromatic surround variance in the "gamut expansion effect." J Vis 8:30. CrossRef Medline

Franks KM, Russo MJ, Sosulski DL, Mulligan AA, Siegelbaum SA, Axel R (2011) Recurrent circuitry dynamically shapes the activation of piriform cortex. Neuron 72:49-56. CrossRef Medline

Friedman HS, Zhou H, von der Heydt R (2003) The coding of uniform colour figures in monkey visual cortex. J Physiol 548:593-613. CrossRef Medline

Gordon J, Shapley R (2006) Brightness contrast inhibits color induction: evidence for a new kind of color theory. Spat Vis 19:133-146. CrossRef Medline

Gordon J, Abramov I, Chan H (1994) Describing color appearance: hue and saturation scaling. Percept Psychophys 56:27-41. CrossRef Medline

Hadjikhani N, Liu AK, Dale AM, Cavanagh P, Tootell RB (1998) Retinotopy and color sensitivity in human visual cortical area V8. Nat Neurosci 1:235-241. CrossRef Medline

Highsmith J, Crognale MA (2010) Attentional shifts have little effect on the waveform of the chromatic onset VEP. Ophthalmic Physiol Opt 30:525533. CrossRef Medline

Hurvich LM, Jameson D (1957) An opponent-process theory of color vision. Psychol Rev 64:384-404. CrossRef Medline

Isaacson JS, Scanziani M (2011) How inhibition shapes cortical activity. Neuron 72:231-243. CrossRef Medline

Jameson D, Hurvich LM (1959) Perceived color and its dependence on focal, surrounding, and preceding stimulus variables. J Opt Soc Am 49:890898. CrossRef Medline

Johnson EN, Hawken MJ, Shapley R (2001) The spatial transformation of color in the primary visual cortex of the macaque monkey. Nat Neurosci 4:409-416. CrossRef Medline

Johnson EN, Hawken MJ, Shapley R (2008) The orientation selectivity of color-responsive neurons in macaque V1. J Neurosci 28:8096-8106. CrossRef Medline

Johnson E, Hawken M, Shapley R (1998) Interactions of chromatic and luminance signals in Macaque V1 neurons. Soc Neurosci Abstr 24:30511.

Kaplan E (2014) The M, P, and K pathways of the primate visual system revisited. In: The new visual neurosciences (Werner JS, Chalupa LM, eds.). Cambridge, MA: Massachusetts Institute of Technology.

Kirschmann A (1891) Ueber die quantitativen Verhältnisse des simultanen Helligkeits- und Farben-contrastes. Philosophische Studien 6:417-491.

Klem GH, Lüders HO, Jasper HH, Elger C (1999) The ten-twenty electrode system of the International Federation. the International Federation of Clinical Neurophysiology. Electroencephalogr Clin Neurophysiol Suppl 52:3-6. Medline

Krauskopf J (1963) Effect of retinal image stabilization on the appearance of heterochromatic targets. J Opt Soc Am 53:741-744. CrossRef Medline

Kulikowski JJ, Robson AG, Murray IJ (2002) Scalp VEPs and intra-cortical responses to chromatic and achromatic stimuli in primates. Doc Ophthalmol 105:243-279. CrossRef Medline

Livingstone MS, Hubel DH (1984) Anatomy and physiology of a color system in the primate visual cortex. J Neurosci 4:309-356. Medline

Li X, Chen Y, Lashgari R, Bereshpolova Y, Swadlow HA, Lee BB, Alonso JM (2014) Mixing of chromatic and luminance retinal signals in primate area V1. Cereb Cortex. Advance online publication. Retrieved Jan. 23, 2014. doi: 10.1093/cercor/bhu002. CrossRef Medline

Martinovic J, Mordal J, Wuerger SM (2011) Event-related potentials reveal an early advantage for luminance contours in the processing of objects. J Vis 11:piil. CrossRef Medline

McKeefry DJ, Zeki S (1997) The position and topography of the human colour centre as revealed by functional magnetic resonance imaging. Brain 120:2229-2242. CrossRef Medline

Mullen KT, Dumoulin SO, McMahon KL, de Zubicaray GI, Hess RF (2007) Selectivity of human retinotopic visual cortex to S-cone-opponent, L/Mcone-opponent and achromatic stimulation. Eur J Neurosci 25:491-502. CrossRef Medline

Murray IJ, Parry NRA, Carden D, Kulikowski JJ (1987) Human visual-evoked potentials to chromatic and achromatic gratings. Clin Vis Sci 1:231-244.

Nakamura M, Kakigi R, Okusa T, Hoshiyama M, Watanabe K (2000) Effects of check size on pattern reversal visual evoked magnetic field and potential. Brain Res 872:77-86. CrossRef Medline

Onofrj M, Fulgente T, Thomas A, Malatesta G, Peresson M, Locatelli T, Martinelli V, Comi G (1995) Source model and scalp topography of pattern reversal visual-evoked potentials to altitudinal stimuli suggest that infoldings of calcarine fissure are not part of VEP generators. Brain Topogr 7:217-231. CrossRef Medline

Rabin J, Switkes E, Crognale M, Schneck ME, Adams AJ (1994) Visualevoked potentials in three-dimensional color space: correlates of spatiochromatic processing. Vision Res 34:2657-2671. CrossRef Medline

Schluppeck D, Engel SA (2002) Color opponent neurons in V1: a review and model reconciling results from imaging and single-unit recording. J Vis 2:480-492. CrossRef Medline

Shapley R, Hawken MJ (2011) Color in the cortex: single- and doubleopponent cells. Vision Res 51:701-717. CrossRef Medline

Shapley RM, Xing D (2013) Local circuit inhibition in the cerebral cortex as the source of gain control and untuned suppression. Neural Netw 37:172181. CrossRef Medline

Souza GS, Gomes BD, Lacerda EM, Saito CA, da Silva Filho M, Silveira LC (2008) Amplitude of the transient visual-evoked potential (tVEP) as a function of achromatic and chromatic contrast: contribution of different visual pathways. Vis Neurosci 25:317-325. CrossRef Medline

Switkes E, Bradley A, De Valois KK (1988) Contrast dependence and mechanisms of masking interactions among chromatic and luminance gratings. J Opt Soc Am A 5:1149-1162. CrossRef Medline

Victor JD, Maiese K, Shapley R, Sidtis J, Gazzaniga MS (1989) Acquired central dyschromatopsia: analysis of a case with preservation of color discrimination. Clin Vision Sci 4:183-196.

Wachtler T, Sejnowski TJ, Albright TD (2003) Representation of color stimuli in awake macaque primary visual cortex. Neuron 37:681-691. CrossRef Medline

Wu GK, Arbuckle R, Liu BH, Tao HW, Zhang LI (2008) Lateral sharpening of cortical frequency tuning by approximately balanced inhibition. Neuron 58:132-143. CrossRef Medline 